\title{
A saúde na percep̧̧ão do adolescente
}

| 'Cléa Adas Saliba Garbin, ²Artênio José Isper Garbin, 35uzely Adas Saliba Moimaz, ${ }^{4}$ Patrícia Elaine Gonçalves I

Resumo: Na fase da adolescência, o indivíduo experimenta os melhores índices de saúde, os quais podem ser mantidos e/ou melhorados, dependendo da percepção e valorização dos mesmos, influenciando na sua qualidade de vida. O estudo avaliou a opinião de adolescentes $(n=493)$ da Fundação Mirim do Município de Araçatuba - SP, quanto à saúde geral e bucal, bem como sua percepção sobre elas, por meio questionário semiestruturado, durante o ano letivo de 2006, já que os mesmos possuem acesso à atenção à saúde bucal e palestras sobre saúde. Dentre os entrevistados 34,5\% conceituaram a saúde como coisa ou algo que precisa(mos) cuidar; 26,6\%, como bem-estar; $18,1 \%$, como a própria existência; e 14,5\%, como ausência de doença. Em relação aos conceitos de saúde bucal, 68,4\% definiram como higiene; 16,2\%, como aparência; e 15,5\%, como ausência de doenças bucais. A percepção desses jovens quanto a sua saúde em geral foi para 46,5\%, ótima, e 44,1\%, boa, enquanto que a da sua saúde bucal foi para $53,6 \%$, boa, e $24 \%$, ótima. Pode-se concluir que os adolescentes têm uma ideia tanto sobre saúde geral como saúde bucal, porém a classificação é menor no que tange à saúde bucal, pois envolve aspectos afetivos, estéticos e sociais. Ademais, percebe-se a necessidade de programas educativo-preventivos direcionados a esse grupo.

> Palavras-chave: Saúde do adolescente; saúde bucal do adolescente; percepção; adolescente.

\author{
${ }^{1}$ Vice-Coordenadora do \\ Programa de Pós-graduação \\ em Odontologia Preventiva e \\ Social da Faculdade de \\ Odontologia de Araçatuba - \\ UNESP. Endereço eletrônico: \\ cgarbin@foa.unep.br \\ 2 Professor no Programa de \\ Pós-graduação em \\ Odontologia Preventiva e \\ Social da Faculdade de \\ Odontologia de Araçatuba - \\ UNESP. \\ ${ }^{3}$ Professora no Programa de \\ Pós-graduação em \\ Odontologia Preventiva e \\ Social da Faculdade de \\ Odontologia de Araçatuba - \\ UNESP. \\ ${ }^{4}$ Aluna de Doutorado do \\ Programa de Pós-graduação \\ em Odontologia Preventiva e \\ Social da Faculdade de \\ Odontologia de Araçatuba - \\ UNESP.
}

Recebido em: 21/11/2007. Aprovado em: 29/09/2008. 


\section{Introdução}

No Brasil, jovens com idade entre 15 a 19 anos correspondem a 15,6\% da população (IBGE, 2006). A infância e a adolescência são períodos do ciclo de vida marcados por grande vulnerabilidade, por representarem fases em que o ser humano está crescendo e se desenvolvendo, tanto física como intelectualmente, e merece atenção redobrada. Por isso, é estratégica e necessária uma educação voltada para a saúde com impacto, que resultará em maior autonomia das pessoas em relação ao cuidado consigo mesmas, com o outro e com o meio em que vivem, para a conquista de melhor qualidade de vida (GUIMARĀES, 2003).

Entre as características psicológicas dos adolescentes, estão a busca de identidade, os desequilíbrios e instabilidades extremas, a alternância de períodos de altivez, introversão, audácia, timidez, descoordenação, urgência, desinteresse e apatia, os conflitos afetivos e as crises religiosas (ABERASTURY; KNOBEL, 1992). Assim, quando uma criança entra no processo de adolescência, questiona de forma radical sua identidade, a dos seus pais, assim como a sociedade em que vive, por serem surpreendentes as mudanças físicas e emocionais que são parte dessa transição. $\mathrm{O}$ período da adolescência adquiriu uma reputação tempestuosa e estressante, o que não é exagerado, tal a importância do processo (BEE, 1997).

$\mathrm{Na}$ fase da adolescência, o indivíduo experimenta os melhores índices de saúde e vitalidade, que o permitirão realizar suas tarefas na idade adulta (PALAZZO et al., 2003). No entanto, é nela que se apresenta um período de risco para a saúde bucal, devido à maior independência em relação ao consumo de alimentação mais açucarada e certa repulsa em relação à higiene bucal, além de outros fatores agregados. Mas sabe-se que as doenças bucais prevalentes podem ser prevenidas com medidas de autocuidado e de proteção específica (VALENTE, 2004). Por isso, é importante realizar estudos com os adolescentes para que, a partir de suas vivências, expectativas e visão do mundo, se possa compreender melhor como se expressam e buscam ajuda, com o objetivo de auxiliar e orientar a criação de medidas mais eficazes, e o planejamento de ações educativopreventivas voltadas para esse grupo (PALAZZO et al., 2003).

O objetivo deste trabalho foi avaliar o conhecimento do adolescente quanto à saúde geral e bucal, bem como sua percepção sobre elas. 


\section{Material e métodos}

O presente trabalho foi submetido e aprovado pelo Comitê de Ética em Pesquisa com Seres Humanos da Faculdade de Odontologia de Araçatuba - UNESP, registrado sob o protocolo $\mathrm{n}^{\mathrm{o}}$. 2006-01243.

A população objeto deste estudo foi composta por 493 adolescentes ( $n=493)$, de ambos os sexos, de 15 a 17 anos de idade, matriculados na Fundação Mirim de Araçatuba - São Paulo, instituição que oferece aos adolescentes cursos de capacitação para o mercado de trabalho. Esse número de indivíduos corresponde à totalidade de alunos da instituição no ano letivo de 2006. A pesquisa foi realizada especificamente na primeira semana de aula, quando os alunos se apresentam à instituição e recebem informaçôes quanto aos cursos. Não houve perda de sujeito de pesquisa.

Para que fosse concretizada a pesquisa, foi enviado a Fundação Mirim de Araçatuba ofício solicitando a colaboração da mesma para a realização da pesquisa, a qual concordou prontamente.

Antes do ingresso do adolescente à instituição, solicitou-se a todos os pais e/ou responsáveis que assinassem o Termo de Consentimento Livre e Esclarecido, no qual se informava que seu filho seria convidado a participar de pesquisa sobre o tema de saúde e saúde bucal. Isso ocorreu pelo fato de os adolescentes serem considerados, segundo a Resolução CONEP/CNS no 196/96, sujeitos de pesquisa vulneráveis, ou seja, não apresentam autonomia suficiente para decidir sobre sua vida. Porém, antes das perguntas propriamente ditas, foi inserido no questionário um breve cabeçalho, esclarecendo aos possíveis adolescentes participantes sobre a voluntariedade da sua participação, a manutenção do sigilo de suas identidades (apesar de não ser solicitada identificação dos respondentes) e a publicação dos dados obtidos. Ademais, foram respeitados todos os ditames do Conselho Nacional de Saúde do Ministério da Saúde.

$\mathrm{O}$ instrumento de análise utilizado foi um questionário semiestruturado, elaborado pelos pesquisadores, o qual apresentava cinco questôes subjetivas e duas objetivas relativas à saúde geral e bucal. Antes de realizar a pesquisa concretamente, o mesmo instrumento foi testado em estudo piloto com adolescentes que não fizeram parte da pesquisa, para adequação do instrumento, quanto à melhora do entendimento das perguntas e para que não ocorresse viés na pesquisa (quadro 1). 


\section{QUESTIONÁRIO}

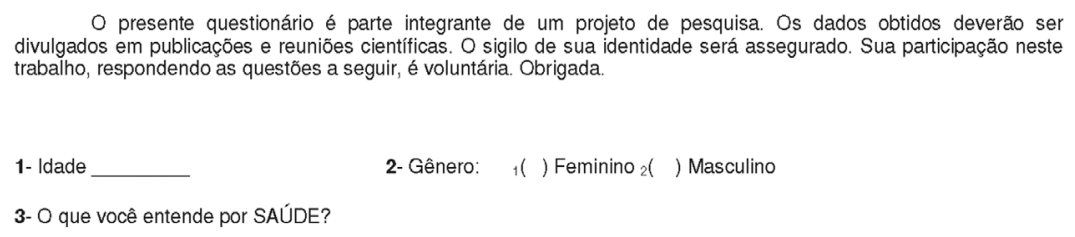

Vale ressaltar que não foi realizada nenhuma atividade educativa prévia à aplicação do questionário visando a obter dados que refletissem o real conhecimento de cada aluno sobre o tema avaliado. A coleta dos dados foi realizada por um único examinador devidamente treinado para a aplicação do instrumento. Os questionários foram respondidos individualmente pelos alunos, em sala de aula, na presença do pesquisador, para que este pudesse fornecer instruçôes sobre seu preenchimento e esclarecer eventuais dúvidas, sem contudo influenciar o conteúdo das respostas.

Após a coleta dos dados, estes foram armazenados em um banco de dados do programa EPI INFO 3.2, sendo que as respostas das perguntas abertas foram analisadas qualitativamente quanto ao seu conteúdo, a partir da Análise de Conteúdo Temática (BARDIN, 1994), e agrupadas em categorias. Já as respostas das perguntas fechadas foram analisadas quantitativamente. Em ambas foi realizada a distribuição de frequências. 


\section{Resultados}

Dentre os pesquisados, $59 \%$ são do gênero feminino e $41 \%$ do masculino. A idade dos mesmos varia entre 14 a 17 anos, sendo que a maioria $(74,4 \%)$ apresenta 15 anos de idade.

Quanto ao tema "saúde", foi perguntado aos adolescentes qual seu entendimento sobre o assunto. As respostas foram distribuídas segundo as categorias (quadro 1). Dentre os entrevistados 34,5\% conceituaram saúde como "coisa ou algo que precisa (mos) cuidar"; 26,6\%, bem-estar; $18,1 \%$, a própria existência; e 14,5\%, ausência de doença.

Quadro 2 - Distribuição da frequência absoluta e relativa das categorias de "Saúde" segundo os adolescentes. Araçatuba - SP, 2007

\begin{tabular}{|c|c|c|}
\hline Categorias & $\mathbf{n}$ & $\%$ \\
\hline $\begin{array}{l}\text { Algo ou coisa que precisa cuidar -“A saúde é uma } \\
\text { coisa que devemos ter muito cuidado, se estiver se } \\
\text { sentindo mal mesmo }\end{array}$ & 154 & 34,5 \\
\hline que não seja nada, vá ao posto." & & \\
\hline $\begin{array}{l}\text { Bem-estar - "É ter o corpo e a mente funcionando } \\
\text { bem em harmonia" }\end{array}$ & 119 & 26,6 \\
\hline Existência - "Sem ela não somos nada" & 81 & 18,1 \\
\hline Ausência de doença - "Saúde é não ter doenças" & 65 & 14,5 \\
\hline $\begin{array}{l}\text { Higiene - "É ter uma boa higiene, é manter-se } \\
\text { sempre limpo" }\end{array}$ & 38 & 8,5 \\
\hline $\begin{array}{l}\text { Direito, propriedade do ser humano - "É o que } \\
\text { todos devem ter direito, mas não é bem assim que } \\
\text { acontece" }\end{array}$ & 35 & 7,8 \\
\hline $\begin{array}{l}\text { Prevenção de doenças - "Saúde é uma resistência, } \\
\text { que evita de pegar doenças" }\end{array}$ & 32 & 7,2 \\
\hline $\begin{array}{l}\text { Condicionamento físico - "É como se encontra o } \\
\text { nosso corpo, o nosso condicionamento físico" }\end{array}$ & 27 & 6,0 \\
\hline Alimentação saudável - "É ter boa alimentação" & 25 & 5,6 \\
\hline Atenção Médica - "Parte que cuida das doenças" & 17 & 3,8 \\
\hline $\begin{array}{l}\text { Qualidade de vida - "Saúde é uma qualidade de } \\
\text { vida" }\end{array}$ & 12 & 2,7 \\
\hline $\begin{array}{l}\text { Outros - "A coisa mais abençoada que Deus nos } \\
\text { deixou" }\end{array}$ & 3 & 0,7 \\
\hline
\end{tabular}

* 46 adolescentes pesquisados não responderam a pergunta 
Em relação aos conceitos de "Saúde Bucal", 68,4\% definiram como higiene; 16,2\%, aparência; e 15,5\%, ausência de doenças bucais (quadro 2).

Quadro 3 - Distribuição da frequência absoluta e relativa das categorias de Saúde Bucal segundo os adolescentes. Araçatuba - SP, 2007

\begin{tabular}{|c|c|c|}
\hline Categorias & n & $\%$ \\
\hline $\begin{array}{l}\text { Higiene - "Saúde Bucal seria você passar o fio dental, } \\
\text { escovar, etc..." }\end{array}$ & 292 & 68,4 \\
\hline $\begin{array}{l}\text { Aparência - "Quando seus dentes estão bons } e \\
\text { bonitos" }\end{array}$ & 69 & 16,2 \\
\hline $\begin{array}{l}\text { Ausência de doenças bucais - "Saúde Bucal é estar } \\
\text { sem cáries e sem outras doenças" }\end{array}$ & 66 & 15,5 \\
\hline $\begin{array}{l}\text { Cuidado profissional - "É estar indo ao dentista } \\
\text { regularmente prevenindo doenças" }\end{array}$ & 54 & 12,6 \\
\hline $\begin{array}{l}\text { Dentes, gengivas saudáveis - "Saúde Bucal é ter os } \\
\text { dentes, a gengiva saudável, sem problemas" }\end{array}$ & 36 & 8,4 \\
\hline $\begin{array}{l}\text { Saúde da boca - "É a saúde de nossa boca, nossos } \\
\text { dentes e todos os demais componentes da boca" }\end{array}$ & 25 & 5,9 \\
\hline $\begin{array}{l}\text { Alimentação saudável - "Devemos ter uma } \\
\text { saudável refeição para não estragar os dentes" }\end{array}$ & 4 & 0,9 \\
\hline
\end{tabular}

Sobre a percepção desses jovens quanto a sua saúde, 46,5\% responderam "ótima” e 44,1\%, "boa" (gráfico 1).

Gráfico 1 - Distribuição da frequência percentual da percepção da saúde segundo os adolescentes. Araçatuba - SP

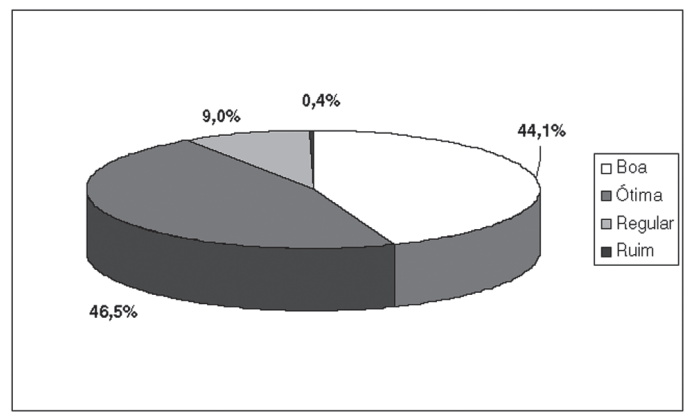

* 1 adolescente pesquisado não respondeu à pergunta 
Já a percepção desses adolescentes quanto a sua saúde bucal, para 53,6\% foi "boa" e 24\%, "ótima”.

Gráfico 2 - Distribuição da frequência percentual da percepção da saúde bucal segundo os adolescentes. Araçatuba - SP

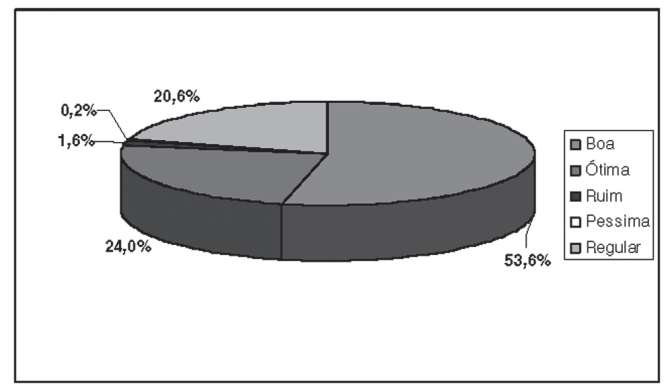

* 6 adolescentes pesquisados não responderam à pergunta

Para relacionar com a saúde, perguntou-se aos adolescentes se os mesmos haviam apresentado alguma doença no último ano. A resposta foi positiva para 124 adolescentes $(25,4 \%)$. Destes, apenas $3(2,4 \%)$ se referiram a alguma doença bucal.

\section{Discussão}

Dentre as categorias de saúde, observa-se que os adolescentes pesquisados apresentaram uma ideia sobre saúde, concordando com Capra (1982), que descreve sua concepção de saúde, que é holística e ecológica, e enfatiza a inter-relação e interdependência essencial de todos os fenômenos.

$\mathrm{O}$ adolescente está exposto a fatores de risco, como sexo inseguro, consumo de álcool, tabaco, drogas, alimentação não-saudável e sedentarismo, os quais possibilitam o aparecimento de problemas de saúde nessa idade e podem se agravar no decorrer do tempo ((ROSA, 2003). Percebe-se, entretanto, a responsabilidade dos mesmos sobre o zelo por sua saúde, que não é só momentânea, mas durante toda a vida, conforme citado por 34,5\% dos entrevistados, que conceituaram saúde como "algo ou coisa que precisamos cuidar".

Segre e Ferraz (1997) enfatizam que a expressão "bem-estar" deva estar ligada à experiência íntima do sujeito, com suas crenças e valores, conforme relatado por $26,6 \%$ dos entrevistados, ou seja, a subjetividade dos mesmos deve ser valorizada. 
De acordo com a Constituição brasileira (BRASIl, 1988), o art. 196 menciona que a Saúde é direito de todos e dever do Estado, o que também foi citado por $7,8 \%$ dos pesquisados, demonstrando o conhecimento dos mesmos quanto a seus direitos como cidadãos brasileiros.

Quanto ao condicionamento físico relatado por 6\% dos adolescentes, compreende-se que o jovem está voltado atualmente para os aspectos estéticos, na comparação do seu corpo a um padrão de beleza. Sabe-se que o desejo de possuir uma boa aparência não é mais encarado como sinal de vaidade, e sim uma necessidade ELIAS et al., 2001).

Ferreira (1998) observou, em seu estudo, que qualquer alteração na qualidade de vida do indivíduo, ou seja, quando o mesmo não consegue trabalhar, comer, dormir ou realizar atividade a que está habitualmente acostumado, implica estar doente, conforme mencionado por alguns jovens (14,5\%) em que a saúde é a ausência de doença.

O termo "qualidade de vida", caracterizado como saúde por 2,7\% dos pesquisados, é frequentemente observado na literatura. No entanto, autores ressaltam que algumas medidas de qualidade de vida relacionada à saúde têm abordagem eminentemente restrita aos sintomas e às disfunções, contribuindo pouco para uma visão abrangente dos aspectos não-médicos associados à qualidade de vida, a qual abrangeria os aspectos físicos, psicológicos e sociais do indivíduo (GLADIS et al., 1999).

Quanto à percepção de saúde, Rosa (2003), em estudo com adolescentes mexicanos, constatou que em geral estes se percebem como saudáveis, e que apenas $2 \%$ deles consideram sua saúde ruim, assim como foi observado em $0,4 \%$ dos pesquisados neste trabalho.

Sobre os adolescentes que haviam apresentado alguma doença no último ano, apenas 2,4\% dos adolescentes citaram a doença bucal. De acordo com Flores e Drehmer (2003), isso ocorre porque, para os adolescentes, a doença cárie é representada pela dor de dente, e não é considerada doença, porque é comum, assim como a gengivite, que é percebida como um desequilíbrio na coloração da gengiva.

Quanto aos conceitos de saúde bucal, 12,6\% dos adolescentes mencionaram o cuidado profissional como tal. Porém, a procura pela atenção odontológica pelos adolescentes está diretamente relacionada à participação e interesse dos responsáveis na manutenção da saúde oral deles, bem como a própria vaidade dos mesmos (COLARES et al., 2002; SEVERO, 2001). 
Mirsrachi e Arellano (1995), em seu estudo com 381 adolescentes chilenos de idade entre 12 a 20 anos, perceberam que a estética é a principal razão para o cuidado bucal, concordando com 16,2\% dos pesquisados, que conceituaram a saúde bucal com a aparência. Para Weyne (1997), no nível psicossocial, uma boca sadia garante a manutenção da boa aparência, da expressão e da comunicação interpessoal, sendo fator da maior importância na preservação de autoestima. Através da face, uma região sempre exposta do corpo humano, a estética bucal comprometida pode-se tornar motivo de ansiedade.

Fernandes (2002), em seu trabalho com adolescentes grávidas e não-grávidas, observou não haver diferença nos comportamentos e crenças em saúde bucal em ambos os grupos. No entanto, a Odontologia não foi prioridade para as adolescentes, apesar de valorizarem muito a estética em seus discursos.

Sobre a percepção da saúde bucal, no Projeto Saúde Bucal Brasil 2003 (BRASIL, 2003), observou-se que $44,6 \%$ dos adolescente classificaram sua saúde bucal como boa, $34 \%$ como regular e $5,8 \%$ como ótima. Neste trabalho foi obtida uma maior porcentagem quanto à classificação boa $(53,6 \%)$ e ótima $(24 \%)$, sendo o item regular $(20,6 \%)$ menor que o encontrado pelo projeto.

Na percepção dos entrevistados, a classificação para sua saúde bucal obteve um valor menor (77,6\%), quando comparado a sua saúde geral (90,6\%). Ou seja, eles acreditam apresentar uma melhor saúde geral do que bucal, devido à maior prevalência da categoria regular de $9 \%$ (saúde) para 20,6\% (saúde bucal). Isto se deve ao fato de a saúde bucal ter agregado aspectos afetivos e estéticos, conforme observado no estudo de Matos e Lima-Costa (2006), em que a melhor autoavaliação da saúde bucal estaria diretamente relacionada ao maior número de visitas ao dentista, necessidade autorreferida de tratamento odontológico, presença de maior número de dentes permanentes, menor número de dentes cariados ou restaurados e melhor condição periodontal (ATCHISON; GIFT, 1997; GIFT et al., 1998; MATTHIAS et al., 1995).

\section{Conclusão}

Frente aos trabalhos analisados e resultados obtidos, pode-se concluir que a maioria dos adolescentes possui uma ideia tanto sobre saúde geral como saúde bucal. A percepção sobre as mesmas demonstrou que a maioria dos jovens tem uma percepção boa ou ótima, porém tornam-se mais rigorosos quanto à percepção da saúde bucal, pois estão envolvidos aspectos afetivos, estéticos e sociais. 
Além disso, observa-se a necessidade de uma estratégia populacional educativopreventiva dirigido a esse grupo, a fim de melhorar seus conceitos e percepção sobre saúde bucal, e a compreensão de que esta apresenta relação direta com a saúde geral do indivíduo.

\section{Referências}

ABERASTURY, A.; KNOBEL, M. A. A adolescência normal. Porto Alegre: Artes Médicas, 1992. ATCHISON, K. A.; GIFT, H. C. Perceived oral health in a diverse sample. Adv Den Res, v. 11, p. 272-280, 1997.

BARDIN, L. Análise de conterido. Lisboa: Persona, 1994.

BEE, H. O ciclo vital. Porto Alegre: Artes Médicas, 1997.

BRASIL. Senado Federal. Constituição da República Federativa do Brasil. Braślia: Senado Federal, 1988.

BRASIL. Ministério da Saúde. Projeto SB Brasil 2003. Condições de saúde bucal da população brasileira 2002-2003: resultados principais. Brasília: Coordenação Nacional de Saúde Bucal, 2003.

CAPRA, F. Oponto de mutação. São Paulo: Cultrix, 1982.

COLARES, V. et al. Avaliação da saúde bucal de adolescentes de Recife-PE-Brasil. Rev Bras Ciênc Saúde, v. 6, n. 3, p. 217-224, 2002.

ELIAS, M. S. et al. A importância da saúde bucal para adolescentes de diferentes estratos sociais do município de Ribeirão Preto. Rev Latino-Am Enfer, v. 9, n. 1, p. 88-95, 2001.

FERNANDES, M. L. M. F. Análise comparativa das percep̧̧ôes em saúde bucal de adolescentes grávidas e não-grávidas: um ponto de partida para a promoção em saúde bucal. Dissertação (Mestrado em Odontologia) - Faculdade de Odontologia, Universidade Federal de Minas Gerais, Belo Horizonte, 2002.

FERREIRA, J. O corpo sígnio. In: MINAYO, M. C. S.; ALVES, P. C. (orgs.). Saúde, doença: um olhar antropológico. Rio de Janeiro: Fiocruz; 1998. p. 98-104.

FLORES, E. M. T. L.; DREHMER, T. M. Conhecimentos, percepçóes, comportamentos e representaçôes de saúde e doença bucal dos adolescentes de escolas públicas de dois bairros de Porto Alegre. Ciênc Saúde Colet, v. 8, n. 3, p. 743-752, 2003.

GIFT, H. C.; ATCHISON, K. A.; DRURY, T. F. Perceptions of the natural dentition in the context of multiple variables. J Dent Res., v. 77, p. 1.529-1538, 1998.

GLADIS, M. M. et al. Quality of life: expanding the scope of clinical significance. JCons Clin Psychol, v. 67, p. 320-331, 1999. 
GUIMARÃES, G. R. A. Promoção da saúde na escola: saúde bucal como objeto de saber. Dissertação (Mestrado em Odontologia) - Faculdade de Odontologia, Universidade Federal do Rio de Janeiro, Rio de Janeiro, 2003.

IBGE. Disponível em: http:/www.ibge.br. Acesso em: 15 maio 2006.

MATOS, D. L.; LIMA-COSTA, M. F. Auto-avaliação da saúde bucal entre adultos e idosos residentes na regiāo Sudeste: resultados do projeto SB- Brasil, 2003. Cad Saúde Pública, v. 22, n. 8, p. 1.699-1.707, 2006.

MATTHIAS, R. E. et al. Factors affecting self-ratings of oral health. J Public Health Den., v. 55, p. 197-204, 1995.

MISRACHI, L. C.; ARELLANO, O. M. Conductas y factores determinantes en salud oral de los adolescentes. Rev Chil Pediat, v. 66, n. 6, p. 317-322, 1995.

PALAZZO, L. S.; BÉRLA, J. U.; TOMASI, E. Adolescentes que utilizan servicios de atención primaria: ¿Cómo viven? ¿Por qué buscan ayuda y cómo se expresan? Cad Saúde Pública, v. 19, n. 6, p. 1.655-1665, 2003.

ROSA, A. C. La salud de adolescentes en cifras. Salud Publica del Méx., v. 45, p. 153-165, 2003.

SEGRE, M.; FERRAZ, F. C. O conceito de saúde. Rev Saúde Pública, v. 31, n. 5, p. 538-542, 1997.

SEVERO, I. F. Abordagem psicológica do adolescente pelos cirurgióes-dentistas da cidade do Recife. Dissertação (Mestrado em Odontologia) - Faculdade de Odontologia, Universidade Federal de Pernambuco, Recife, 2001.

VALENTE, M. S. G. Adolescência y salud bucal. Adolesc Latinoam., v. 98, n. 1, p. 170-174, 2004.

WEYNE, S. C. A Construção do paradigma de promoção de saúde: um desafio para as novas gerações. In: KRIGER, L. (org.). Promoção de saúde bucal. São Paulo: Artes Médicas; 1997. p. 1-26. 


\section{Abstract}

\section{Health in the adolescent's viewpoint}

During adolescence, the individual experiences the best health indexes, which can be maintained and/or improved, depending on their perception and valorization influencing their quality of life. The study assessed the opinion of adolescents $(n=493)$ from the Mirim Foundation of the Araçatuba county in São Paulo State, Brazil, about their oral and general health, as well as their perception about them, through a semistructured questionnaire, in the school year period of 2006 , since they have access to information on oral health care and speeches about health in general. Among the interviees, $34.5 \%$ referred to health as something to be cared of; $26.6 \%$ defined it as well-being;ç $18.1 \%$, as related to their very existence, and $14.5 \%$, as an absence of disease. In relation to the concepts of oral health, $68.4 \%$ defined it as hygiene; $16.2 \%$, as physical appearance and $15.5 \%$, as to the absence of oral diseases. The perception of these youngsters about their health in general was excellent for $46.5 \%$, and good for $44.1 \%$. Concerning their oral health, for $53.6 \%$ it was good, and for $24 \%$, excellent. One can conclude that the adolescents have an idea about general health, as well as oral health, but the oral health classification is lower, since it involves emotional, esthetic and social aspects. Moreover, the need of prevention-educative programs designed for this group is indubitable.

> Key words: teen's health; oral health of adolescents; perception; adolescent. 